\title{
Preclinical Evaluation of Pharmaceutical Compositions and Their Components for Urinary Health
}

\author{
Francesco De Seta ${ }^{1,2}$, Jonathan Wayne Lowery ${ }^{3}$, Oladipupo Ogunbekun OMS II ${ }^{3}$, Bryan Larsen ${ }^{3 *}$ \\ ${ }^{1}$ Institute for Maternal and Child Health "IRCCS Burlo Garofolo", Trieste, Italy \\ ${ }^{2}$ Department of Medicine, Surgery and Health Sciences, University of Trieste, Trieste, Italy \\ ${ }^{3}$ Marian University, Indianapolis, IN, USA \\ Email: ^blarsen@marian.edu
}

How to cite this paper: De Seta, F., Lowery, J.W., Ogunbekun, O. and Larsen, B. (2020) Preclinical Evaluation of Pharmaceutical Compositions and Their Components for Urinary Health. Open Journal of Obstetrics and Gynecology, 10, 151-165. https://doi.org/10.4236/ojog.2020.1010013

Received: December 13, 2019

Accepted: January 10, 2020

Published: January 13, 2020

Copyright $\odot 2020$ by author(s) and Scientific Research Publishing Inc. This work is licensed under the Creative Commons Attribution International License (CC BY 4.0).

http://creativecommons.org/licenses/by/4.0/

\begin{abstract}
Background: The need for products that treat or prevent urinary tract infections without resort to antibiotics that may select for resistant bacterial strains has created a need to develop antibiotic-free therapeutics. Objective: To conduct an exploratory evaluation of intrinsic antimicrobial activity of a panel of compounds alone or in combination against urinary pathogens and probiotic organisms as well as their effect on biofilm formation and immune activity in infected cultured bladder epithelial cells. Results: Of the compounds tested, $1 \%$ citric acid was most consistently inhibitory to urinary pathogens as were combinations containing citric acid. Two combinations of compounds were tested and both showed antimicrobial activity when challenged with $1 \times$ $10^{5}$ bacteria/mL. The two combination products and D-mannose did not inhibit probiotic microorganisms and one composition increased the inhibitory potential of probiotic organisms. Results obtained with biofilm studies were variable, but probiotic biofilm was enhanced under some circumstances. Biofilms of some urinary pathogens were reduced by antimicrobial mixtures. A strong cytokine response was elicited when T24 bladder epithelial cells were infected for one hour with urinary pathogens and a modest reduction in cytokine response was recorded with some combinations of test compounds. Conclusion: Citric acid alone and mixtures of various compositions containing citric acid inhibited the growth and biofilm formation by 4 uropathogens. Probiotic organisms grew in the presence of mannose and citric acid-containing combinations. One of the combinations enhanced probiotic activity of Lactobacilli against uropathogens. When infecting T24 cell monolayers with uropathogens, potential modulation of inflammatory activity was demonstrated.
\end{abstract}




\section{Keywords}

Urinary Pathogen, Antibacterial, Inflammation, Bladder Epithelial Cells

\section{Introduction}

Urinary tract infections are among the commonest of primary care issues accounting for millions of physician visits annually, and can range from asymptomatic to acute and may extend upward to involve the kidneys and spread beyond leading to sepsis and death in compromised individuals. Antibiotic treatment is standard but challenges and opportunities remain for progress in dealing with this prevalent medical problem including dosing or alternatives [1]. Antimicrobial pharmaceuticals may be limited either by antibiotic resistance or may select for antibiotic resistant strains. Recurrence of urinary infection with or without resistance development is concerning [2] and a growing number of patients have an interest in using natural products, which may be conveniently obtained over the counter. Interest in non-antibiotic approaches encompasses acute cystitis and prophylaxis of recurrent urinary infection [3].

Current approaches to recurrent urinary infections in women have been described in detail in a recent review [4] and emphasize a more expansive approach to this problem than just focusing on antimicrobials. The role of physical concerns such as anatomic anomalies, or urinary calculi, behavioral components of risk and lack of meta data support for some non-antibiotic approaches, lead ultimately to the presentation of a selection of antibiotic agents that are used for prophylaxis or intermittent treatment. Thus, it appears that antibiotics will retain a role for the time being despite the fact that clinicians are increasingly cautious about their overuse.

Beerpoot and co-workers [5] [6] provided reviews of clinical uses of non-antibiotic approaches to urinary prophylaxis, mentioning various categories of intervention including vaccination, probiotic Lactobacillus, estriol and cranberry. Mannose has support from basic and clinical studies as a bacterial adherence-blocking reagent [7] [8] [9] but a Latin American consensus group indicated mannose has less research support than bacterial lysates or local estrogen [10]. Numerous other, less studied, examples lack confirmation of efficacy [11]. Presented here are preclinical findings using a panel of compounds alone or in combination to inhibit urinary pathogens. Combinations of these compounds were selected based on anticipated potential usefulness in acute cystitis or prophylaxis against recurrent infection. However, these combinations were not marketed therapeutics. Of interest were bacterial growth, bacterial biofilm formation and possible immune reactions in the bladder.

\section{Methods}

The laboratory work described here was conducted at the Marian University 
College of Osteopathic Medicine between January and May of 2019.

\subsection{Microorganisms}

All bacterial isolates used in this study were obtained from American Type Culture Collection and regardless of their original source, should be considered as laboratory strains because they were not recently in a human host. Specifically, these included E. coli ATCC 11775 [12], Proteus mirabilis ATCC 7002 [13], Streptococcus agalactiae ATCC13813 [14] and Enterococcus faecalis ATCC19433 [15].

Probiotic microorganisms were from frozen laboratory stocks and included the following Lactobacillus species: crispatus, gasseri, jensenii, and rhamnosis as well as Saccharomyces boulardii. All cultures were stored at $-80^{\circ} \mathrm{C}$ and grown overnight in brain heart infusion broth (BHI) before inoculation into experiments.

\subsection{Test Materials}

Except for common laboratory chemicals (citric acid and potassium carbonate) all compounds used in determining antimicrobial, antibiofilm and cytokine testing, were supplied by Giellepi S.p.A (Milan, IT) and were designated as follows: fibergum B (Batch 180728), propolis (Propol G Lot 19115), arabinogalactan (Lot FA16186), D-mannose (Lot DM180521), Dry Cranberry (Lot PF0907041018), Dry Nettle Leaf 4:1 (Lot P60042201), Dry Dandelion 4:1 (Lot N6243701), Polydextrose Litesse (Lot 1922415008), Astragalus CHNQ.A (Lot 813475).

Each test material was made to the desired concentration in either purified water or BHI and autoclaved before use in growth studies. Some of these compounds were not entirely soluble at the desired concentration, but were introduced into experiments as sterilized suspensions.

Two combinations prepared as two-fold concentrates to allow for dilution in experiments, were studied as well as individual components of the combinations. Combinations were designated as Composition A (D-mannose $4 \%$, potassium carbonate $3 \%$, citric acid $3 \%$, astragalus $0.6 \%$, nettle $0.16 \%$ ) and Composition $\mathrm{B}$ (D-mannose 4\%, Polydextrose 2\%, Arabinogalactan 0.6\%, Fibergum 0.4\%, Propolis 0.4\%, Dandelion 0.1\%).

These compositions are abbreviated respectively as A and $\mathrm{B}$. Where D-mannose is used alone it is abbreviated in text and figures as $\mathrm{M}$ and these abbreviations are used consistently throughout.

\subsection{Growth Studies}

Unless otherwise noted, bacterial growth studies to demonstrate inhibition followed the basic pattern of minimal inhibitory concentration determination. Serial two-fold dilutions of the test article (compound or A or B) were made in $\mathrm{BHI}$ in 96 or 48 -well plates, inoculated with test bacterium at approximately $1 \times$ 
$10^{4} \mathrm{CFU} / \mathrm{mL}$, grown at $37^{\circ} \mathrm{C}$ overnight and read in a spectrophotometric plate reader at $450 \mathrm{~nm}$ with starting absorbance subtracted.

\subsection{Biofilm}

Biofilm studies were conducted in 24-well plate (allowing for $2 \mathrm{~mL}$ culture per well). Each well was inoculated heavily with $100 \mathrm{uL}$ of overnight culture of the test bacterium into $2 \mathrm{~mL}$ of compound or composition. Plates were sealed with parafilm to retard evaporation and incubated for 7 days at $37^{\circ} \mathrm{C}$.

After development of biofilm, cultures were aspirated and wells washed 3 times with purified water and then $1 \%$ safranin [16] was added to each well for 30 minutes to stain the biofilm. The safranin was aspirated and wells washed 3 times with purified water and allowed to dry. Dye was mobilized with alcohol and transferred to a 96-well plate and Abs $562 \mathrm{~nm}$. Absorbance is proportional to biofilm biomass.

\subsection{Cell Culture}

T24 bladder cancer cells [17] were obtained from ATCC and stored in liquid nitrogen until use. They were propagated in 6-well plates with $5 \mathrm{~mL}$ of McCoy's medium supplemented with $10 \%$ fetal bovine serum at $37^{\circ} \mathrm{C}$ in $5 \% \mathrm{CO}_{2}$. They were grown to confluence prior to infection.

Infections using uropathogens were made at an approximate bacterial concentration of $1 \times 10^{5} \mathrm{CFU} / \mathrm{mL}$. Infection occurred for 1 hour before aspirating the infected medium and replacement with fresh sterile medium. Cultures were returned to the incubator overnight and supernatant fluids were recovered and sterilized with $0.22 \mathrm{uM}$ syringe filter.

\subsection{Cytokine Markers}

Relative cytokine and chemokine levels in spent T24 cultures were determined by chemiluminescent spot Western blot assays according to the manufacturer's directions (R\&D systems).

Quantiation of human inflammatory cytokines and human chemokines employed the $\mathrm{BD}$ diagnostics cytokine bead array systems (cytokine and chemokine tested separately) which was used with the BD Acuri C6 cytometer. The manufacturer's protocol was followed; analysis employed the FCAP software supplied by Becton Dickinson.

\section{Results}

\subsection{Antibacterial Activity}

As an initial screen for antimicrobial activity, $1 \% \mathrm{w}: \mathrm{v}$ of each of the compounds was incorporated into BHI medium with $1.5 \%$ agar and test organisms were spotted on the surface of the agar. Only citric acid and cranberry provided inhibition and because of the incompatibility of the largely insoluble cranberry powder with subsequent assays, cranberry was not investigated further. 
To determine if the compositions noted above had antibacterial activity A and $\mathrm{B}$ were prepared in $\mathrm{BHI}$ and diluted and inoculated with the test bacteria. $\mathrm{D}$-mannose was a major component in $\mathrm{A}$ and $\mathrm{B}$ and thus, D-mannose (M) was also included in this experiment. The starting material was prepared as a 2 -fold concentrated solution so equal parts inhibitor and BHI would be equivalent to the full-strength inhibitor. The results for each of the test organisms were similar ( $40 \%$ coefficient of variation of absorbance readings between bacterial species) indicating that the Gram negative and Gram positive uropathogens responded similarly to the compositions. Enterococcus showed weaker growth in BHI, so the biggest contributor to the $\mathrm{CV}$ was due to this organism; however its trend mirrored the other three uropathogens. These results are presented in Figure 1. Of note, under these conditions, $\mathrm{M}$ appeared to have some suppression of bacterial growth. The absence of citric acid in B may have contributed to its diminished activity compared to A. Also apparent in this experiment was the dose-response relationship including an effect of D-mannose, which may have been due to high osmolality.

From each of the test conditions a $10 \mathrm{uL}$ aliquot of culture was spotted on agar medium and the A composition showed loss of viability, but the B and mannose (M) did not provide a microbicidal effect in 24 hours.

\subsection{Probiotic and Prebiotic Conditions}

A hazard of suppressing bacterial growth of uropathogens either by use of antibiotics or non-antibiotic inhibitors is the potential detrimental effect on probiotic organisms. In this experiment 5 probiotic organisms were challenged with A, B, M and compared to growth in BHI. Again, the compositions were prepared

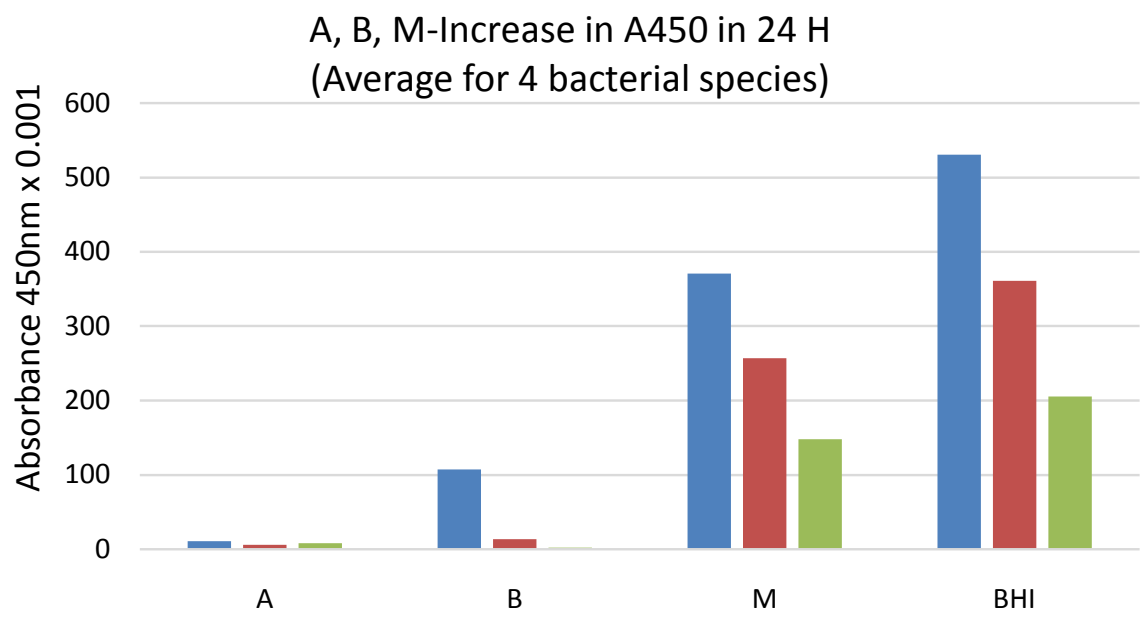

Figure 1. Average absorbance change (from pre-inoculation absorbance) at 24 hours A and $\mathrm{B}$ compared to $\mathrm{M}$ and control growth medium. The concentrations evaluated are indicated by the colors. Blue $50 \%(\mathrm{~A}, \mathrm{~B}$ or $\mathrm{M})$ and $50 \%$ BHI. Orange is $75 \% \mathrm{~A}, \mathrm{~B}$ or $\mathrm{M}$ and $25 \%$ BHI. Gray is $90 \% \mathrm{~A}, \mathrm{~B}$, or M with $10 \% \mathrm{BHI}$. (Mannose was at $4 \%$ concentration in all three test conditions before dilution with $\mathrm{BHI}$; no mannose was present in BHI). BHI was included in all conditions to ensure that low bacterial counts were not due to nutrient deficiency although the effect of nutrient dilution was seen in the BHI only condition. 
at double strength to be combined with an equal volume of BHI and the BHI control was diluted with an equal volume of sterile water. The results obtained are presented in Figure 2.

After 24 hours incubation the data show the B furnished growth equal to or greater than the BHI control medium. L. gassseri and L. jensenii seemed poorly supported by BHI. However, even for composition A, growth of all but one organism ( $S$. boulardii) showed greater growth than in BHI. A possible explanation for this finding is that A contains citric acid and the Lactobacilli may demonstrate acid tolerance. The effect of composition B might be the result of prebiotic compounds in the test article. $\mathrm{M}$ did not have a consistent effect as it gave similar or slightly less growth compared to BHI for all probiotic organisms except $L$. gasseri for which it apparently supported increased growth.

Taken together, these findings suggest that neither A nor B represent a hazard for these probiotic organisms within the context of this in vitro experiment.

While it is useful to know whether probiotic organism growth is stimulated or inhibited in various media, it is more relevant to determine how much probiotic activity they may exert under these conditions. Probiosis may be gauged by growing the probiotic organism under suitable conditions, recovering the supernatant fluid and challenging with a test organism.

For A, B and M, Lactobacilli were grown for 48 hours along with BHI without any potential prebiotic additive. The ratio of the test compositions ( $\mathrm{A}, \mathrm{B}$ and $\mathrm{M}$ were 9 parts to one part $\mathrm{BHI}$ and the $\mathrm{BHI}$ control contained 9 parts water to one part BHI. Culture supernatant fluid was filtered to remove bacteria and then challenged with each of the 4 uropathogens. The results obtained are presented in Figure 3, which indicates the 24 hour growth in the Lactobacillus-conditioned culture supernatants. As with the earlier experiments, the uropathogens showed

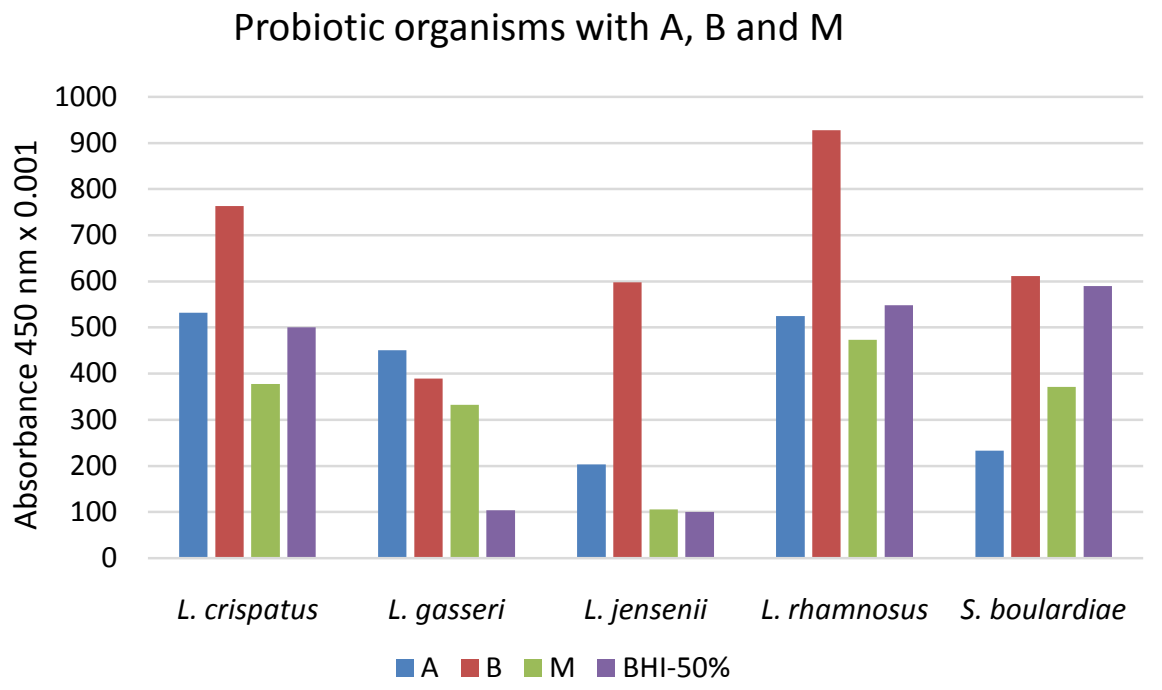

Figure 2. Growth of probiotic organisms in the presence of equal volumes of double strength A, B or M and BHI. BHI controls are equal volumes of full strength BHI and sterile water. Inoculum was a 1:10,000 dilution of an overnight culture of each of the test organism. 


\section{Conditioned Media Challenged with UTI Bacteria}

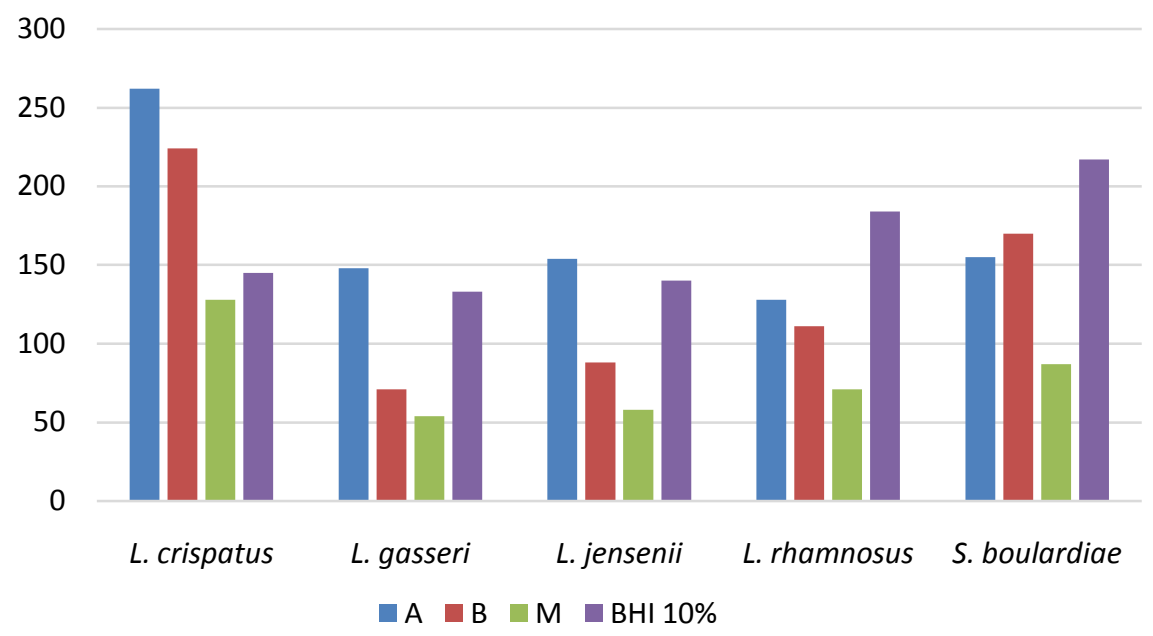

Figure 3. The probiotic organisms indicated were grown in A, B, M and BHI. Sterilized supernatants of the spent cultures re-inoculated with uropathogens were incubated for 24 hours. Each bar indicates the average for the 4 organisms.

similar trends in response to the conditioned medium and averages for the organisms are presented. For each of the Lactobacilli, the B furnished slightly more probiotic activity than A. This is not surprising as the B had compounds that are expected to exert prebiotic activity. Also of note, the $\mathrm{M}$ resulted in uniformly probiotic conditions; however it must be recognized that there could be a significant carryover of mannose in the spent cultures. A chemical analysis would confirm this but was not part of this study.

\subsection{Biofilm}

Biofilms are an essential part of pathogenic processes of infectious microorganisms but also a part of the process of stabilizing probiotic populations. Hence the need to understand the effects of potential urinary treatments was applied to both pathogenic and probiotic organisms.

The ability of uropathogens to develop biofilm in the presence of the multi-component $\mathrm{A}$ and $\mathrm{B}$ were evaluated for each organism. Because $\mathrm{M}$ was a common constituent of A and B, it was also tested along with BHI alone. Additives prepared double strength and mixed with equal volumes of BHI before inoculation with uropathogens. As shown in Figure 4, A developed very little biofilm and coupled with the information on growth inhibition activity by A, it is likely that this reflects limitation on growth as much as an independent effect on biofilm. For E. coli, both mannose and B reduced by about half the amount of biofilm compared to the BHI control. However, for the other microbial species the effect was minimal.

While support for biofilm suppression was not obtained for B or M, the ability to enhance biofilm of probiotic organism was an opportunity explored in the following experiment. As with the uropathogens, the panel of probiotic organisms 


\section{Biofilm from Uropathogens}

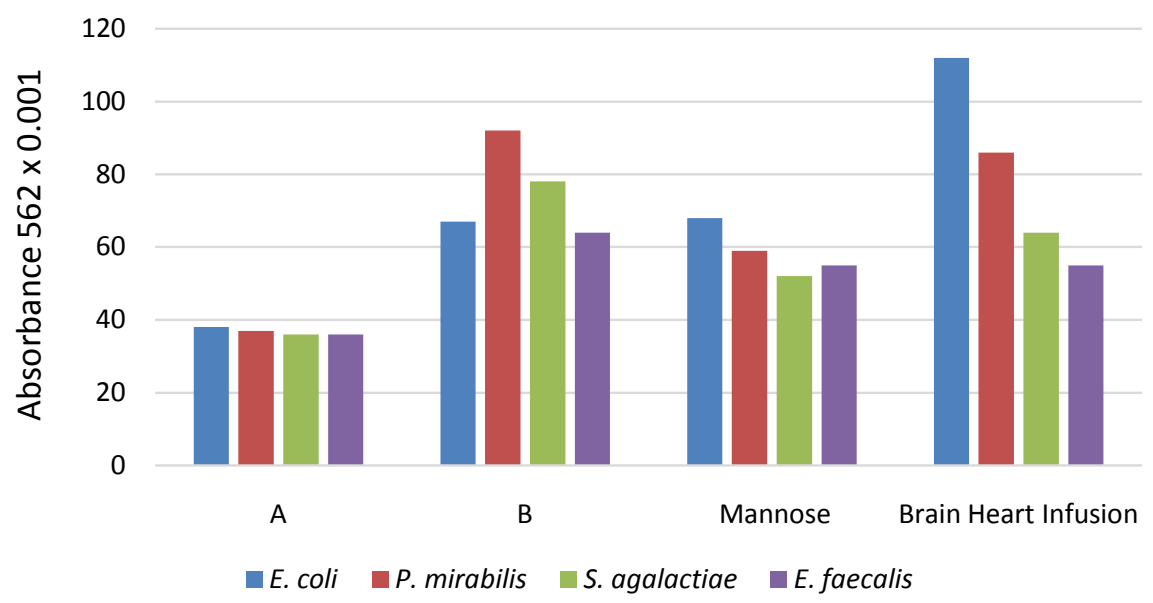

Figure 4. Biofilm production by 4 bacterial species.

were grown in A, B and M. In addition, polydextrose at a concentration equal to that in $\mathrm{B}$ was also tested. Again, the additives were prepared double strength and combined with equal volumes of BHI. The result is shown in Figure 5. In this experiment, B was more supportive of biofilm formation and D-mannose and polydextrose alone did not increase biofilm.

An additional observation from this experiment, especially in light of the effect of A on uropathogen biofilm was that biofilm was not decreased by A which suggests that if this were applied in vivo it may be compatible with the beneficial flora.

\subsection{Bladder Epithelial Integrity and Reactivity}

T24 bladder cancer cells allowed a variety of experiments involving bacterial infection of cell cultures and the relationship of the compositions being studied to the responses to in vitro infection. The first question to be examined was the effect on healthy bladder epithelial cells upon exposure to A and B as well as M. An exposure time of 4 hours was selected and not surprisingly, A appeared to be the harshest treatment as shown in Figure 6. Propidium iodide staining, which results in red fluorescence, did not indicate any treatment yielded a significant loss of viability. There may be some loss of confluence among monolayers, but mainly with C-B. D-mannose alone may have had some osmotic effect.

With the availability of the T24 cells and confidence that the A and B were compatible with cell survival, the ability to use inflammatory markers to further investigate the effects of uropathogens as well as the effect of therapies on the cells was investigated. The proteome profiling system for human cytokines and chemokines was used as a scouting tool. This chemiluminescent dot Western blot system provides relative quantitation of 13 biomarkers. Each experiment has 4 membranes limiting the experiment to 3 test conditions and one control. A limited experiment was performed in which T24 cells were exposed overnight to A, B and $\mathrm{M}$ with controls consisting of fresh culture medium. A second set of 


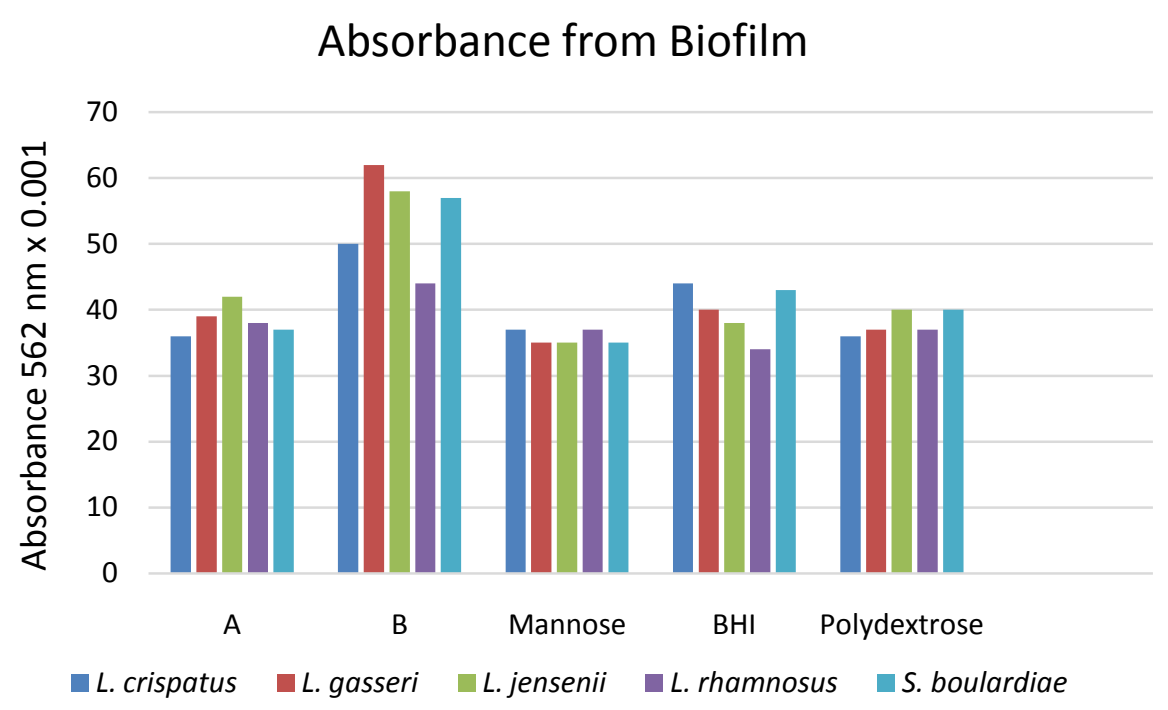

Figure 5. The ability of additives to BHI to affect the amount of biofilm produced by probiotic species.
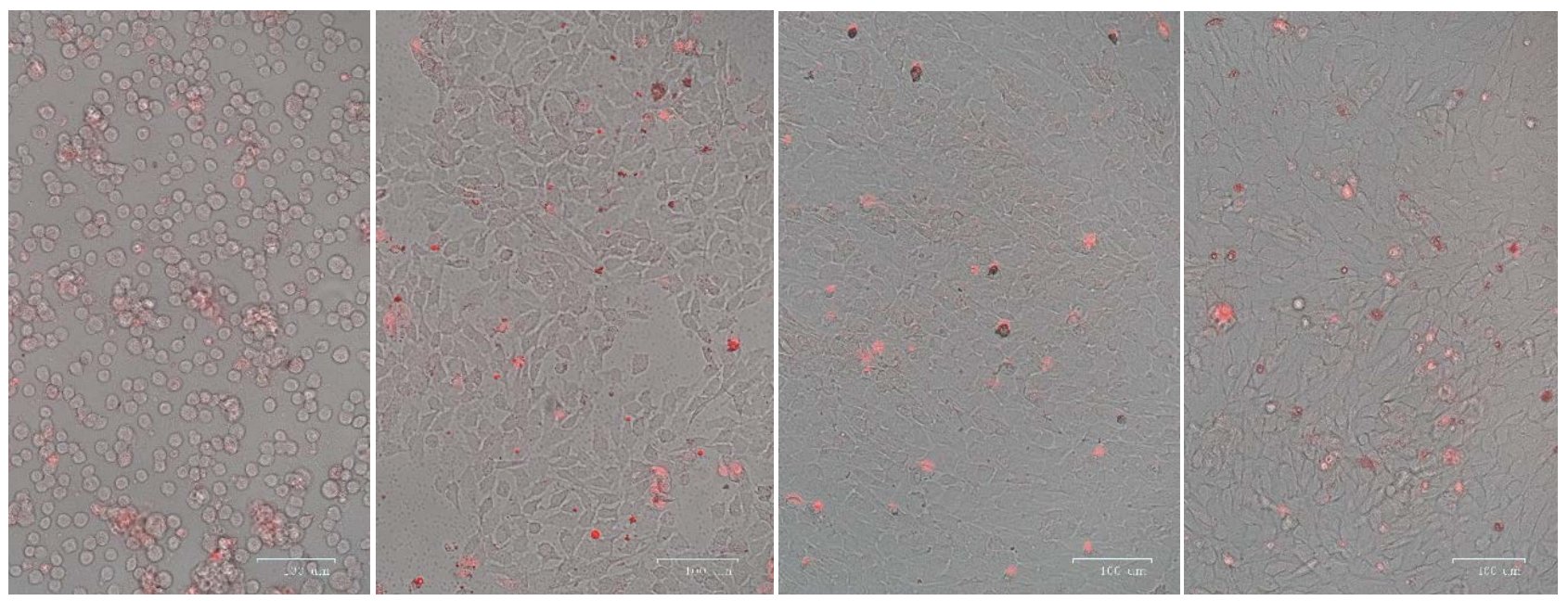

Figure 6. The appearance of T24 bladder cancer cells (400×, markers are $100 \mathrm{uM}$ ) after four hours exposure to A, B, and M (left to right) with untreated bladder epithelial cells with phosphate buffered saline. All cultures were stained with propidium iodide.

membranes used cell culture supernatant fluids infected with E. coli, S. agalactiae and $E$. faecalis with a control consisting of fresh culture medium.

A 24 hour exposure time was selected because information was lacking on whether biomarkers would have accumulated to detectable levels during shorter incubations. And even if the cell culture should die off during the 24 hour incubation, the released cytokines should still be available for detection.

Results from this experiment were based on chemiluminescent signal intensity wherein control membrane signals could be categorized as fold-intensity increase over the control sample. Responses were categorized as indicated in Table 1 and presented as a heat map. From this evaluation, it was immediately clear that a far stronger biomarker response occurred with bacteria versus the compositions or D-mannose. 
Table 1. Cytokine profiling membrane heat map. Relative cytokine signal is presented as fold increase or decrease compared to signal from control (consisting of T24 cells refed with fresh media and incubated as for additives or uropathogens). Values are categorized from purple to red on the basis of ratio of test to control: Red is $\geq 3$, orange $\geq 2$, yellow $\geq$ 1 , green $\geq 0.8$, blue $\geq 0.6$, purple $\geq 0.1$. Empty cells had no signal detected.

\begin{tabular}{ccccccc}
\hline Cytokine & $\mathrm{A}$ & $\mathrm{B}$ & $\mathrm{M}$ & E. coli & S. agalactiae & E. faecalis \\
\hline CCL2 & & & & & 0.872 & \\
CCL5 & & & & & 0.303 & \\
CXCL1 & 0.889 & 0.359 & 0.802 & 4.202 & 0.906 & 3.137 \\
CXCL10 & & & & 0.559 & 0.987 & 0.294 \\
GCSF & 0.132 & 0.816 & 1.136 & 1.586 & 0.999 & 1.439 \\
GMCSF & 0.282 & 0.463 & 1.311 & 1.139 & 0.942 & 1.138 \\
ICAM1 & & & & 0.057 & & 0.042 \\
IL1ra & & & & 0.070 & & \\
IL1 beta & & & & 0.818 & & 0.572 \\
IL6 & 0.734 & 0.401 & 0.773 & 1.728 & 0.744 & 1.540 \\
IL8 & 0.997 & 0.599 & 0.878 & 3.021 & 0.602 & 2.833 \\
MIF & 1.408 & 1.215 & 0.365 & 1.087 & 0.684 & 1.272 \\
SERPIN & 1.623 & 1.206 & 0.539 & 1.068 & 1.102 & 1.171 \\
\hline
\end{tabular}

An alternative cytokine assay used a fluorescent detection bead array analyzed by flow cytometry. Because some of the biomarkers detected in the membrane array were also detected in the bead array and the bead array could accommodate more tests, a more extensive evaluation of bacterial infection with and without treatment was undertaken. It was also found that 24 hours of incubation resulted in cytokine levels that were out of range for the bead assay parameter. Thus, bacterial infection occurred for 1 hour followed by 3 washes with fresh medium. Infected and treated cultures were harvested at 4 hours after infection. Assays were quantitative with results expressed in $\mathrm{pg} / \mathrm{mL}$ and because of the dynamic range for these assays, results were presented as $\log \mathrm{pg} / \mathrm{mL}$ (Table 2). It appeared that many cytokines were high even without infection and this may have been due to the stress of the sham manipulation ( 3 changes of fresh medium).

This experiment, however suggests there is an opportunity to lower the inflammatory response in the bladder cell culture and suggests that A or B or some of the potentially anti-inflammatory compounds we included in this research could be used in this manner.

A study was conducted to determine if A, B or M could alter the expression of key cytokines in uropathogen-infected T24 cell cultures. Although 6 cytokines were in the bead array panel, TNF, IL12-70p and IL10 were not detected at meaningful levels and are not presented in the results below. Note that because of the smaller range in values, these cytokine concentrations are not presented as log concentrations. After a 1 hour exposure to urinary organisms and 3 washes the 
Table 2. Effect of infection on T24 bladder epithelial cells at 4 hours post infection. Cytokine values are expressed as log pg/mL. Relative concentrations: yellow $\geq 2$ fold increase over uninfected; orange $>5$ fold increase; green $\leq$ half of control.

\begin{tabular}{cccccc}
\hline Cytokine & Uninfected & Escherichia coli & $\begin{array}{c}\text { Proteus } \\
\text { mirabilis }\end{array}$ & $\begin{array}{c}\text { Streptococcus } \\
\text { agalactiae }\end{array}$ & $\begin{array}{c}\text { Enterococcus } \\
\text { faecalis }\end{array}$ \\
\hline Il1 beta & 0.6 & 0.91 & 1.07 & 0.92 & 0.49 \\
Il6 & 4.41 & 4.50 & 4.50 & 4.3 & 4.4 \\
TNF & 1.09 & 0.75 & 1.02 & 1.33 & - \\
Il8 & 4.93 & 4.48 & 4.43 & 4.5 & 4.4 \\
IP10 & 3.57 & 3.66 & 3.69 & 3.71 & 3.73 \\
RANTES & 2.02 & 2.23 & 2.85 & 2.36 & 2.51 \\
MCP & 2.72 & 3.02 & 3.1 & 3.01 & 3.27 \\
\hline
\end{tabular}

results and sampling 6 hours thereafter, bead array data were obtained, assembled and presented below. It is clear, as already discovered, that there is some basal level of cytokine production, especially in the case of IL6 and IL8, which appear even in the absence of infection.

A more significant finding is that A and B have cytokine suppressing effects and the magnitude of these effects in some cases are quite profound. The $\mathrm{M}$ effects were somewhat more variable. In Table 3, cytokine production was most affected for IL6 and IL8 and despite differences in compositions both had effects on cytokines, although IL1-beta showed a somewhat divergent response.

Table 3. Cytokine absolute concentrations determined by bead array flow cytometry of T24 cell supernatants.

\begin{tabular}{ccccccccccccccc}
\hline \multicolumn{1}{c}{ Interleukin 1 beta: pg/mL } & \multicolumn{3}{c}{ Interleukin 6: pg/ $\mathrm{mL}$} & \multicolumn{3}{c}{ Interleukin 8: pg/mL } \\
\hline Treatment & None & A & B & M & None & A & B & M & None & A & B & M \\
\hline Organism & & & & & & & & & & & & & \\
Not Infected & 47 & 4.3 & 4.7 & 11,500 & 6760 & 9317 & 10,680 & 16,050 & 15,940 & 4350 & 13,550 & 22,938 \\
E. coli & 2.12 & 33.7 & 0 & 11,429 & 13,949 & 1110 & 984 & 30,490 & 21,568 & 5100 & 866 & 21,367 \\
P. mirabilis & 3.17 & 25.9 & 8.87 & 1.02 & 14,542 & 495 & 1839 & 12,217 & 20,882 & 267 & 1704 & 17,067 \\
S. agalactiae & 0.72 & 86.48 & 4.49 & 3.1 & 11,208 & 282 & 1455 & 12,167 & 15,870 & 413 & 1152 & 17,486 \\
E. faecalis & 0.18 & 3 & 2.17 & 0.55 & 10,574 & 66 & 103.7 & 13,300 & 21,360 & 362 & 58 & 2316 \\
\hline
\end{tabular}

\section{Discussion}

Urinary tract infections, especially in adult women are a continuing problem of great magnitude and in addition to the symptoms of uncomplicated urinary tract infection, complicated infections can have substantial morbidity especially in elderly and compromised individuals. A further complexity related to urinary infection is that repeated antibiotic treatment necessitated by multiple episodes enhances the likelihood of antibiotic resistance development. 
Academic and pharmaceutical researchers are continuing to seek non-antibiotic alternatives for treatment and for recurrence prevention. The approaches represent many strategies including immune stimulants, probiotics, adhesion blockers, vaccines, hyperimmune bovine colostrum, estrogen, and modulators of symptoms such as NSAIDS [18]. Popular interest in botanical and other natural products such as cranberry or vitamins attracts public as well as scientific interest [19].

Against this backdrop, two proposed combinations of compounds with putative biological properties that may have relevance to urinary health were studied. We elected to obtain data on these two compositions with the expectation that if interesting biological activities were present, factorial studies could be conducted in a separate and more focused manner. In parallel, some major components of these compositions were investigated independently, specifically $M$ present in both preparations and citric acid which is a major component of $\mathrm{A}$.

The present research was concerned first with the ability of the A and B to inhibit microbial growth with a focus on two Gram negative organisms and two Gram positive organisms. The inhibitory activity discovered was most associated with $\mathrm{A}$ and appeared largely derived from citric acid. While the immediate tendency is to attribute inhibitory activity to the acidity of citric acid, a secondary attribute of citric acid is metal chelation. It is not a strong chelator, but synergy with acidity is a consideration. A companion experiment determined whether the compositions had an inhibitory effect on potentially probiotic organisms which, perhaps due to their acid tolerance, were less affected by A.

Relevant to these findings are studies that indicate acid can induce stress responses in microorganisms that increase survival under adverse conditions [20] [21]. This could account for the relative refractoriness of probiotic organisms to A, but should be applicable to E. coli and Enterococcus as well.

Biofilm represents a challenging area for study, because logically if microorganisms are prevented from growing, the production of biofilm is unlikely. Biofilms of pathogens may be detrimental whereas biofilms of probiotic organisms may be beneficial. The experiments here provide evidence for reduced biofilm in uropathogens exposed to B. In the case of probiotic organisms, B supported approximately $20 \%$ more biofilm than standard growth medium. While these findings do not indicate that the compositions may be useful clinically, they do not detract from further development.

The third major area of investigation employed bladder epithelial cells and their interactions with bacteria and potential therapeutics. We were able to demonstrate that these cells tolerated exposure to the A and B, but not without some alteration to cellular morphology. Inflammatory and stress cytokines were observed in uninfected bladder epithelial cells but we did not determine the basis for this expression. Possible explanations include the manipulation of the cells (removal from incubation conditions, removing and replacing medium and the possibility that fetal bovine serum in the medium caused some reactivity).

An additional feature of the compositions tested was the addition of botanical materials, which may have biological activity as immune modulators. For exam- 
ple, Astragalus was recently reported to attenuate inflammatory and oxidative stress reactions through the NFkB pathway [22]. Dandelion extract modulates TNF expression induced by LPS [23] and in a recent review [24] nettle is described as having numerous bioactive components with varied activities. These and other individual components may be individually assessed in the bladder epithelial cultures describe here.

The bladder epithelial cultures provided a valuable platform for many potential experiments including, but not limited to, titration of bacterial load, timing of infection, testing different bacterial species and strains with varying displays of virulence factor and factorial evaluation of individual components of the A and $\mathrm{B}$. While the various experimental conditions that might be tested are numerous, the limitation is the cost of cytokine analysis making it necessary to limit the work to the experiments deemed most likely to be informative.

Because this research was an exploratory project, not all of the variables were studied, but the results obtained help establish information that suggests how refined and optimized future studies may be carried out. Consideration was given to the possibility that one of the compositions might offer antimicrobial activity and the other might enhance immune or probiotic protection. A better approach for the future may be a single composition incorporating the most beneficial properties of the two compositions. However, this will require additional factorial studies of individual components. A future investigation should approach the matter of a single multipurpose combination.

The overall key findings in this paper suggest that A has antimicrobial activity against uropathogens we tested but is somewhat more aggressive with respect to bladder epithelial cells. B appeared less potent in antimicrobial activity but more supportive of probiotic organisms and having modest modulation of inflammatory activity compared to cell cultures exposed to bacteria.

Apart from the preclinical observations made, it is important to be mindful of the other practical aspects of this work. If the compositions studied are to have clinical relevance, it will be necessary to envision how they could be formulated and how they will be delivered to the site of infection and what the clinical efficacy and tolerability might be. Nevertheless, the work done here establishes a platform on which testing of other potential urinary anti-infective anti-inflammatory compounds or combinations of compounds might be given preliminary evaluation.

\section{Disclosure}

This work was supported by a contract from Giellepi S.p.A, Milan Italy to Bryan Larsen who was responsible for the research plan and execution of the laboratory work carried out at Marian University. The other authors took part in the discussion of the work during the course of the investigation and took part in reviewing and editing the final manuscript. Dr. De Seta provides medical advisor service to Giellepi S.p.A. Combinations used in this research do not represent products of Giellepi S.p.A. 
This work is original and has not been published elsewhere.

\section{Conflicts of Interest}

The authors declare no conflicts of interest regarding the publication of this paper.

\section{References}

[1] Wawrysiuk, S., Naber, K., Rechberger, T. and Miotla, P. (2019) Prevention and Treatment of Uncomplicated Lower Urinary Tract Infections in the Era of Increasing Antimicrobial Resistance-Non-Antibiotic Approaches: A Systemic Review. Archives of Gynecology and Obstetrics, 300, 821-828. https://doi.org/10.1007/s00404-019-05256-Z

[2] Alsubaie, S.S. and Barry, M.A. (2019) Current Status of Long-Term Antibiotic Prophylaxis for Urinary Tract Infections in Children: An Antibiotic Stewardship Challenge. Kidney Research and Clinical Practice, 38, 441-454. https://doi.org/10.23876/j.krcp.19.091

[3] Kim, H.W., Chung, D.H., Kim, S.A. and Rhee, M.S. (2019) Synergistic Cranberry Juice Combinations with Natural-Borne Antimicrobials for the Eradication of Uropathogenic Escherichia coli Biofilm within a Short Time. Letters in Applied Microbiology, 68, 321-328. https://doi.org/10.1111/lam.13140

[4] Dason, S., Dason, J.T. and Kapoor, A. (2011) Guidelines for the Diagnosis and Management of Recurrent Urinary Tract Infection in Women. Canadian Urological Association Journal, 5, 316-322. https://doi.org/10.5489/cuaj.11214

[5] Beerepoot, M. and Geerlings, S. (2016) Non-Antibiotic Prophylaxis for Urinary Tract Infections. Pathogens, 5, pii: E36. https://doi.org/10.3390/pathogens5020036

[6] Beerepoot, M.A., Geerlings, S.E., van Haarst, E.P., van Charante, N.M. and ter Riet, G. (2013) Nonantibiotic Prophylaxis for Recurrent Urinary Tract Infections: A Systematic Review and Meta-Analysis of Randomized Controlled Trials. Journal of Urology, 190, 1981-1989. https://doi.org/10.1016/j.juro.2013.04.142

[7] Duncan, D. (2019) Alternative to Antibiotics for Managing Asymptomatic and Non-Symptomatic Bacteriuria in Older Persons: A Review. British Journal of Community Nursing, 24, 116-119. https://doi.org/10.12968/bjcn.2019.24.3.116

[8] Russo, E., Montt Guevara, M., Giannini, A., Mannella, P., et al. (2019) Cranberry, D-Mannose and Anti-Inflammatory Agents Prevent Lower Urinary Tract Symptoms in Women Undergoing Prolapse Surgery. Climacteric, 1-5. https://doi.org/10.1080/13697137.2019.1679110

[9] Milandri, R., Maltagliati, M., Bocchialini, T., Del Prete, C., et al. (2019) Effectiveness of D-Mannose, Hibiscus sabdariffa and Lactobacillus plantarum Therapy in Prevention of Infectious Events Following Urodynamic Study. Urologia, 86, 122-125. https://doi.org/10.1177/0391560318798291

[10] Haddad, J.M., Ubertazzi, E., Cabrera, O.S., Medina, M., et al. (2019) Latin American Consensus on Uncomplicated Recurrent Urinary Tract Infection 2018. International Urogynecology Journal. https://doi.org/10.1007/s00192-019-04079-5

[11] Gill, C.M., Hughes, M.A. and La Plante, K.L. (2019) A Review of Nonantibiotic Agents to Prevent Urinary Tract Infections in Older Women. Journal of the American Medical Directors Association, pii: S1525-8610(19)30379-2.

https://doi.org/10.1016/j.jamda.2019.04.018

[12] Wadley, T.D., Jenjaroenpun, P., Wongsurawat, T., Ussery, D.W., et al. (2019) Com- 
plete Genome and Plasmid Sequences of Escherichia coli Type Strain ATCC 11775. Microbiology Resource Announcements, 8, pii: e00046-19. https://doi.org/10.1128/MRA.00046-19

[13] Minogue, T.D., Daligault, H.E., Davenport, K.W., Bishop-Lilly, K.A., et al. (2014) Draft Genome Assemblies of Proteus mirabilis ATCC 7002 and Proteus vulgaris ATCC 49132. Genome Announcements, 2, pii: e01064-14. https://doi.org/10.1128/genomeA.01064-14

[14] Bashir, S., Sial, R.N., Ashraf, M.U., Mushtaq, M.N., et al. (2018) Phytochemical Analysis and Antimicrobial Activity of Aqueous and Methanolic Extracts of the Flowers of Nelumbium speciosum. Pakistan Journal of Pharmaceutical Sciences, 31, 2429-2434.

[15] Huycke, M.M., Joyce, W. and Wack, M.F. (1996) Augmented Production of Extracellular Superoxide by Blood Isolates of Enterococcus faecalis. The Journal of Infectious Diseases, 173, 743-746. https://doi.org/10.1093/infdis/173.3.743

[16] Ommen, P., Zobek, N. and Meyer, R.L. (2017) Quantification of Biofilm Biomass by Staining: Non-Toxic Safranin Can Replace the Popular Crystal Violet. Journal of Microbiological Methods, 141, 87-89. https://doi.org/10.1016/j.mimet.2017.08.003

[17] Legros, N., Ptascheck, S., Pohlentz, G., Karch, H., et al. (2019) PapG Subtype-Specific Binding Characteristics of Escherichia coli towards Globo-Series Glycosphingolipids of Human Kidney and Bladder Uroepithelial Cells. Glycobiology, 29, 789-802. https://doi.org/10.1093/glycob/cwz059

[18] Whiteside, S.A., Dave, S., Reid, G. and Burton, J.P. (2019) Ibuprofen Lacks Direct Antimicrobial Properties for the Treatment of Urinary Tract Infection Isolates. Journal of Medical Microbiology, 68, 1244-1252. https://doi.org/10.1099/jmm.0.001017

[19] Head, K.A. (2008) Natural Approaches to Prevention and Treatment of Infections of the Lower Urinary Tract. Alternative Medicine Review, 13, 227-244.

[20] Papadimitriou, K., Alegría, A., Bron, P.A., de Angelis, M., et al. (2016) Stress Physiology of Lactic Acid Bacteria. Microbiology and Molecular Biology Reviews, 80, 837-890. https://doi.org/10.1128/MMBR.00076-15

[21] Wang, W., He, J., Pan, D., Wu, Z., et al. (2018) Metabolomics Analysis of Lactobacillus plantarum ATCC 14917 Adhesion Activity under Initial Acid and Alkali Stress. PLoS ONE, 13, e0196231. https://doi.org/10.1371/journal.pone.0196231

[22] Adesso, S., Russo, R., Quaroni, A., Autore, G., et al. (2018) Astragalus membranaceus Extract Attenuates Inflammation and Oxidative Stress in Intestinal Epithelial Cells via NF- $\kappa \mathrm{B}$ Activation and Nrf2 Response. International Journal of Molecular Sciences, 19, 800. https://doi.org/10.3390/ijms19030800

[23] Hu, G., Wang, J., Hong, D., Zhang, T., Duan, H., et al. (2017) Effects of Aqueous Extracts of Taraxacum officinale on Expression of Tumor Necrosis Factor-Alpha and Intracellular Adhesion Molecule 1 in LPS-Stimulated RMMVECs. BMC Complementary and Alternative Medicine, 17, 38. https://doi.org/10.1186/s12906-016-1520-3

[24] Kregiel, D., Pawlikowska, E. and Antolak, H. (2018) Urtica spp.: Ordinary Plants with Extraordinary Properties. Molecules, 23, 1664.

https://doi.org/10.3390/molecules23071664 